\title{
Clinical characteristics and outcome of 55 cases of hepatoblastoma relapses
}

Wei-ling Zhang, Hui-min Hu, Yi-zhuo Wang, Yi Zhang, Jing Li, Yuan Wen, Tian Zhi, Ya-nan Gao, Dong-sheng Huang

Department of Paediatrics, Beijing Tongren Hospital, Capital Medical University, Beijing, China

Submitted: 15 July 2020

Accepted: 14 January 2021

Arch Med Sci

DOI: https://doi.org/10.5114/aoms/132445

Copyright $\odot 2021$ Termedia \& Banach

\section{Abstract}

Introduction: This study aimed to analyse and summarize the clinical characteristics and prognosis of children with hepatoblastoma (HB) recurrence. Material and methods: From January 2009 to June 2018, the clinical data of 55 children with $\mathrm{HB}$, who had recurrence after complete remission (CR), were collected and analysed.

Results: Of the 55 patients, the median duration of follow-up was 65 months, the median interval from CR to the first recurrence was 3 months, and the event-free survival (EFS) times were $42.00 \pm 4.72$ months. For sites of recurrence, 24 patients had lung recurrence only, 6 patients had liver recurrence only, 18 patients had recurrence in both the liver and lung, and 7 patients had recurrence in other sites of the body ( 6 cases of brain and 1 case of mediastinum). The overall 5 -year survival rate of 55 cases was $47.4 \%$ and the 5 -year disease-free survival rate was $37.3 \%$. According to the treatment after recurrence, the patients were divided into the combined treatment group (chemotherapy combined with surgery, 41 cases) and the chemotherapy alone group (14 cases). The 3 -year overall survival rates were $75.2 \%$ and $17.1 \%$, respectively, with statistical difference $\chi^{2}=28.441, p<$ 0.001 . The EFS time and the 3 -year EFS rate of the 2 groups was $55.04 \pm 4.10$ months vs. $32.63 \pm 4.34$ months; $70 \%$ vs. $55.7 \%$, with significant difference $\chi^{2}=3.328, p=0.0068$.

Conclusions: The prognosis of recurrent HB is poor. The main sites of recurrence were lung and liver. After recurrence, chemotherapy combined with surgery can improve prognosis, and complete remission can be achieved.

Key words: hepatoblastoma, metastatic, recurrence, prognosis.

\author{
Corresponding author: \\ Dong-sheng Huang \\ Department of Paediatrics \\ Beijing Tongren Hospital \\ Capital Medical University \\ West South Road 2 \\ Yizhuang Economic and \\ Technological \\ Development Zone \\ Daxing District \\ Beijing 100176, China \\ Phone: (010)58266699, \\ 58266032 \\ Eail: dongshengh53@163. \\ com
}

\section{Introduction}

Hepatoblastoma (HB) is the most common primary malignant liver tumour in children, accounting for more than $1 \%$ of paediatric cancer cases [1]. HB usually occurs before the age of 3 years. The incidence of $\mathrm{HB}$ has steadily increased over the last 3 decades. Between 2006 and 2010, according to US Surveillance, Epidemiology and End Results data, the incidence rates were 9.4 cases per million children $<1$ year of age and 5.5 cases per million children 1-4 years of age, with markedly reduced rates beyond the age of 5 years [2]. The reason for this increasing incidence remains unknown; however, the increased survival rate of premature infants with very low birth weight related to HB is thought to be a possible 
reason [3]. In Japan, children with a birth weight of under $1000 \mathrm{~g}$ are 15 times more likely to develop $\mathrm{HB}$ than normal birth weight children [4], and other data have confirmed that the incidence of $\mathrm{HB}$ in premature infants with very low birth weight is high [5]. Younger age at diagnosis appears to confer a superior prognosis; conversely, the risk for adverse prognostic events rises continuously with increasing age. So the importance of age was a prognostic factor for the outcome of patients with $\mathrm{HB}$ [6].

Semeraro et al. have reported a multicentre retrospective study and found that recurrence cases accounted for approximately $12 \%$ of HB cases. Local recurrence of the liver was found in 21 (36\%) cases, pulmonary metastasis and recurrence were found in 32 (55\%) cases, and recurrence in other sites in the body was found in $5(9 \%)$ cases. Recurrence in other sites included the peritoneum $(n=4)$ and the central nervous system $(n=1)$ [7]. The interval of local recurrence of the liver was shorter (10 months), and the interval of the recurrence of other sites was longer (20 months) [7].

With the development of neoadjuvant chemotherapy and surgery, the overall survival rate of $\mathrm{HB}$ has reached approximately $70 \%[8,9]$. Positive PRE-Treatment EXTent of tumour (PRETEXT) annotation factors are important for the risk stratification [10]. These include V (all 3 hepatic veins and/ or intrahepatic inferior vena cava are involved); $P$ (the left and right portal veins are involved); E (continuous extrahepatic tumour spreading areas, e.g. transverse diaphragm membrane, adjacent organs); $\mathrm{F}$ (multifocal tumour); R (tumour rupture); $M$ (distant metastasis, usually in the lung); and low $\alpha$-foetal protein (AFP) levels (< $100 \mathrm{ng} / \mathrm{ml}$ or $100-1000 \mathrm{ng} / \mathrm{ml}$, indicating that AFP level is increased in infants). Patients in PRETEXT class IV aged 3-7 years have a poor prognosis, and in all PRETEXT classes, patients over 8 years old have worse prognoses than younger patients $[11,12]$. When tumours are responsive, chemotherapy can be used to render recurrent patients resectable, so successful treatment of recurrent $\mathrm{HB}$ relies largely on surgical resection [13]. Outcomes of the Patients With Relapsed Hepatoblastoma Enrolled on Children's Oncology Group (COG) Phase I and II Studies showed that new approaches were needed to better investigate promising new agents for incorporation into treatment strategies, and that standardized response criteria were needed [14].

So, the treatment of advanced and recurrent $\mathrm{HB}$ remains difficult. A study reported that the recurrence rate of HB was $11 \%(59 / 524)$ and found liver and lung to be the most common sites of $\mathrm{HB}$ recurrence (53/59); the main factors associated with a good outcome were PRETEXT group I-III at diagnosis, a high AFP level at relapse, and relapse treatment including both chemotherapy and surgery [7]. A study on the recurrence of $\mathrm{HB}$ in the lung alone indicated that pulmonary tumour resection improved prognosis [15]. The prognosis of recurrent $\mathrm{HB}$ is poor $[16,17]$. In the International Childhood Liver Tumours Strategy Group (SIOPEL) study 1-3, 59 children with recurrent HB had the best results if the tumour was suitable for surgery; the 3-year disease-free survival rate of children receiving chemotherapy and surgery was $34 \%$, and the overall survival rate was only $43 \%$ [7]. Because the incidence of $\mathrm{HB}$ is low and recurrence is rare, more analysis and summary of recurrence cases are needed. In this study, the clinical characteristics and outcome of 55 cases of $\mathrm{HB}$ recurrence were analysed, and the high-risk factors affecting prognosis were analysed and summarized.

\section{Material and methods}

\section{Clinical data}

Detailed clinical data of 55 cases of $\mathrm{HB}$ with recurrence after complete remission (CR) admitted to the Department of Pediatrics, Beijing Tongren Hospital Affiliated to Capital Medical University from 1 January 2009, to 30 June 2018 were collected. All the patients were accurately diagnosed with HB by pathology of excised liver tumour tissue samples. All the patients met the diagnostic criteria of a recurrent case, and the criteria of $\mathrm{HB}$ recurrence included the following: histologically confirmed, definite radiographic evidence and serum AFP increased for 3 consecutive periods in 4 weeks [18]. The patients were divided into 2 categories based on histological characteristics: epithelial type (foetal type, embryonic type, mixed epithelial type, small cell undifferentiated type, macrotrabecular, and cholangioblastoma type) and mixed epithelial and mesenchymal type $[19,20]$.

\section{Classification and grouping}

According to PRETEXT classification and the COG stage system before treatment, the patients were staged [21, 22], and grouped according to the risk factors of initial diagnosis [23].

\section{Therapeutic methods}

The combined treatment programs were mainly chemotherapy and surgery according to a previous procedure [18]. The chemotherapy regimens were mainly those recommended by the consensus of Chinese multi-disciplinary experts in the diagnosis and treatment of HB in China [18]. The specific chemotherapy regimens were C5VD (cisplatin $90 \mathrm{mg} / \mathrm{m}^{2}$ Day 1 + 5-fluorouracil $300 \mathrm{mg} / \mathrm{m}^{2}$ 
Day $2-3$ + vincristine $1.5 \mathrm{mg} / \mathrm{m}^{2}$ Day $1+$ pirarubicin $25 \mathrm{mg} / \mathrm{m}^{2}$ Day 2-3), PLANDO (cisplatin $80 \mathrm{mg} / \mathrm{m}^{2}$ Day 1,8 + pirarubicin $30 \mathrm{mg} / \mathrm{m}^{2}$ Day 8,9$)$, and ICE (ifosfamide $1.5 \mathrm{~g} / \mathrm{m}^{2}$ Day $1-3+$ carboplatin $450 \mathrm{mg} / \mathrm{m}^{2}$ Day 1 + etoposide $100 \mathrm{mg} / \mathrm{m}^{2}$ Day $1-3)$. If the chemotherapy regimen was not effective, it was replaced by the following regimen: (pirarubicin $25 \mathrm{mg} / \mathrm{m}^{2}$ on Day $1-3+$ cyclophosphamide $800-1,000 \mathrm{mg} / \mathrm{m}^{2}$ Day 1 + cisplatin $20 \mathrm{mg} / \mathrm{m}^{2}$ Day 1-5), (pirarubicin $25 \mathrm{mg} / \mathrm{m}^{2}$ Day $1-3$ + etoposide $100 \mathrm{mg} / \mathrm{m}^{2}$ Day $1-4$ + cisplatin $20 \mathrm{mg} / \mathrm{m}^{2}$ Day 1-5), (ifosfamide $300 \mathrm{mg} / \mathrm{m}^{2}$ Day 1-2 + pirarubicin $25 \mathrm{mg} / \mathrm{m}^{2}$ Day $4-5$ + etoposide $100 \mathrm{mg} / \mathrm{m}^{2}$ Day $1-3$ + carboplatin $400 \mathrm{mg} / \mathrm{m}^{2}$ Day 3), (vincristine $1.5 \mathrm{mg} / \mathrm{m}^{2}$ Day $1+$ irinotecan $50 \mathrm{mg} / \mathrm{m}^{2}$ Day 1-5 + cyclophosphamide $250 \mathrm{mg} /$ $\mathrm{m}^{2}$ Day $2-4+$ cisplatin $20 \mathrm{mg} / \mathrm{m}^{2}$ Day $\left.1-5\right)$. Because of the toxicity of doxorubicin, we used pirarubicin instead of doxorubicin in the regime of C5VD.

Multiple recurrence cases were treated with bevacizumab. A time period of 21-28 days was regarded as a chemotherapy cycle. A serum AFP test, routine blood test, biochemical routine test, and electrocardiogram were conducted every cycle, and the primary and metastatic lesions were evaluated every 2 chemotherapy cycles. The operation procedures included resection of tumours in the liver and lungs. The family members of the children were informed and provided informed consent for all examinations and treatments. The study was approved by the Medical Ethics Committee of Beijing Tongren Hospital.

\section{Determination of therapeutic effect and prognosis}

Therapeutic effects were determined according to the following criteria [18]: For completely remission (CR): all target lesions disappeared completely. The physical examination and computed tomography (CT) or magnetic resonance imaging (MRI) revealed that the tumours had disappeared completely, and AFP was normal for more than 4 weeks. The normal range of AFP varies with age. If AFP increases 3 times in a month, it is defined as an increase. For partial remission (PR), tumours were reduced by $\geq 50 \%$, there was no evidence of new onset or disease progression. For stable disease (SD), tumours were reduced by $<50 \%$, and there was no evidence of tumour enlargement or new lesions. For disease progression (PD), tumours increased by $\geq 25 \%$, or there were new tumours, or AFP levels increased. Recurrence (1) was confirmed by biopsy, (2) there was clear imaging evidence, and the serum AFP level increased 3 times within 4 weeks. The deadline for follow-ups was 31 December 2019, or the time that the child died. The survival time was subsequently calculated.

\section{Ethics}

Ethics approval Number: TRECKY2019-033.

\section{Statistical analysis}

Statistical analysis of the data was conducted using statistical software SPSS 21.0. Measurement data were expressed as mean \pm standard deviation $(x \pm S D)$. Count data were expressed as percentages (\%) and were compared between the groups using a $\chi^{2}$ test. The Kaplan-Meier method was used for prognosis analysis, and the survival rate curve was drawn. Univariate analysis of prognosis was conducted using a Log-rank test. High-risk factors affecting prognosis were analysed using a Cox regression analysis. $P<0.05$ was considered statistically significant.

\section{Results}

\section{Clinical characteristics}

Of the 55 cases of recurrent $\mathrm{HB}$, the median age of onset was 20 months (1-1-141 months), and the median interval from $C R$ to the first recurrence was 3 months (1-12 months). One-month-old children with PRETEXT stage III at first diagnosis were treated with 2 cycles of preoperative chemotherapy $\left(C 5 V D^{*} 2\right)$. The tumour was resected when it was significantly reduced after chemotherapy. Children with epithelial embryonic type were treated with 4 cycles of chemotherapy (C5VD*4) after surgery. After stopping treatment for 4 months, children with lung relapse alone were treated with 4 cycles of chemotherapy (PLANDO*2, ICE*2). Metastasis resection was performed when the lung lesion was reduced, and 3 cycles of chemotherapy were performed after surgery to reach complete remission (ICE*3). The median AFP value from CR to the first recurrence was $650 \mathrm{ng} / \mathrm{ml}(27-28,000 \mathrm{ng} / \mathrm{ml})$. Of the 55 cases, 24 had lung recurrence alone, 6 had liver recurrence alone, 18 had recurrence in both the liver and lung ( 3 had recurrence simultaneously in both the liver and lung, and the remainder had recurrence in both the liver and lung successively), and 7 had recurrence in other parts. The resection margin of the 6 liver recurrences alone showed that 3 cases were negative and 3 cases were positive, and the PRETEXT stage of these patients were 5 cases of stage IV and 1 case of stage III at the time of initial diagnosis. The 6 cases were given liver mass resection after neoadjuvant chemotherapy. There were 4 cases given liver surgery twice, 1 case given liver surgery 3 times, and 1 case given liver surgery once. After recurrence, there were between 0 and 33 chemotherapy cycles, and the median number of chemotherapy cycles was 11 . The clinical characteristics of the 55 cases of recurrent $\mathrm{HB}$ are shown in Table I. 
Table I. Clinical features of 55 children with recurrent hepatoblastoma

\begin{tabular}{|c|c|}
\hline Clinical characteristics & Cases $(n, \%)$ \\
\hline \multicolumn{2}{|l|}{ Gender: } \\
\hline Male & $37,67.27$ \\
\hline Female & $18,32.73$ \\
\hline \multicolumn{2}{|l|}{ Age [years]: } \\
\hline$<3$ & $38,69.09$ \\
\hline$\geq 3$ & $17,30.91$ \\
\hline \multicolumn{2}{|l|}{ Pathological types: } \\
\hline Epithelial type: & $37,67.27$ \\
\hline Pure foetal type & $1,1.82$ \\
\hline Embryonal type & $4,7.27$ \\
\hline Mixed epithelial type & $32,58.18$ \\
\hline Small cell undifferentiated type & 0 \\
\hline Giant beam type & $3,5.45$ \\
\hline Mixed epithelium and mesenchyme & $18,32.73$ \\
\hline \multicolumn{2}{|l|}{ PRETEXT stages at initial diagnosis: } \\
\hline Stage III & 11,20 \\
\hline Stage IV & 44,80 \\
\hline \multicolumn{2}{|l|}{ COG stages at initial diagnosis: } \\
\hline Stage III & $23,41.82$ \\
\hline Stage IV & $32,58.18$ \\
\hline \multicolumn{2}{|l|}{ Type of liver tumour: } \\
\hline Unifocal & $34,61.82$ \\
\hline Multifocal & $21,38.18$ \\
\hline \multicolumn{2}{|l|}{ Lung metastasis: } \\
\hline Existing at initial diagnosis & $32,58.18$ \\
\hline Not existing at initial diagnosis & $16,29.09$ \\
\hline Type of lung metastasis: & 55 \\
\hline Unifocal & $2,3.64$ \\
\hline Multifocal & $53,96.36$ \\
\hline \multicolumn{2}{|l|}{ Recurrent: } \\
\hline 1 time & $28,50.91$ \\
\hline 2 times and more & $27,49.09$ \\
\hline
\end{tabular}

\begin{tabular}{|c|c|}
\hline Clinical characteristics & Cases $(n, \%)$ \\
\hline AFP was present at the first relapse: & 55 \\
\hline$<100 \mathrm{ng} / \mathrm{ml}$ & $1,1.82$ \\
\hline$\geq 100 \mathrm{ng} / \mathrm{ml}$ & $54,98.18$ \\
\hline \multicolumn{2}{|l|}{ Treatment after recurrence: } \\
\hline Chemotherapy combined with surgery & $41,74.55$ \\
\hline Chemotherapy alone group & $14,25.45$ \\
\hline \multicolumn{2}{|l|}{ Recurrence of different sites: } \\
\hline Liver alone & $6,10.91$ \\
\hline Lung alone & $24,43.64$ \\
\hline Liver and lung & $18,32.73$ \\
\hline Brain & $6,10.91$ \\
\hline Lung and brain & $5,9.09$ \\
\hline Brain alone & $1,1.82$ \\
\hline Lung and mediastinum & $1,1.82$ \\
\hline \multicolumn{2}{|l|}{ Chemotherapy cycles after recurrence: } \\
\hline$\leq 6$ cycles & $14,74.55$ \\
\hline$>6$ cycles & $41,25.45$ \\
\hline $\begin{array}{l}\text { Chemotherapy results of the first lung } \\
\text { recurrence alone: }\end{array}$ & 24,100 \\
\hline Complete remission & $5,20.83$ \\
\hline Partial remission & $13,54.17$ \\
\hline Stable disease & $1,41.67$ \\
\hline Disease progression & $5,20.83$ \\
\hline Prognosis of lung recurrence alone: & 24,100 \\
\hline Complete remission & $17,70.83$ \\
\hline Death & $7,29.17$ \\
\hline Prognosis of liver recurrence alone: & 6,100 \\
\hline Complete remission & $1,16.67$ \\
\hline Death of HB & 3,50 \\
\hline Death for other reason & $2,33.33$ \\
\hline \multicolumn{2}{|l|}{ Prognosis of all recurrence cases: } \\
\hline Death & $27,49.09$ \\
\hline Complete remission & $27,49.09$ \\
\hline Partial remission & $1,1.82$ \\
\hline
\end{tabular}

PRETEXT - Pretreatment Extension, COG - Children's Oncology Group, AFP - $\alpha$-fetoprotein.

\section{Prognosis analysis}

All patients were followed-up until 31 December 2019. The duration of the follow-up was 12-150 months, and the median duration of follow-up was 65 months. Of the 55 children, 27 died (1 died of myelodysplastic syndrome, who received the same chemotherapy regimen at first diagnosis and relapse periods, though the HB tumour was completely relieved). Twenty-eight children survived; 27 of them achieved CR, and 1 achieved PR. The patient with PR was diagnosed at the age of 6.5 years and relapsed after CR for one month. The patient had a venous tumour thrombus and her lung lesions were not ful- ly controlled. She had been receiving chemotherapy and was still alive when we reported. The survival time was $65.61 \pm 5.77$ months. The overall 3-year survival rate was $61.1 \%$, and the overall 5 -year survival rate was $47.4 \%$ (Figure 1). For the 55 children, the event-free survival times were $42.00 \pm 4.72$ months, and the 3- and 5-year event-free survival rates were $58.2 \%$ and $37.3 \%$, respectively (Figure 2).

\section{Prognosis analysis of different sites of recurrence}

The patients were divided into 4 groups according to the different sites of recurrence: the lung re- 


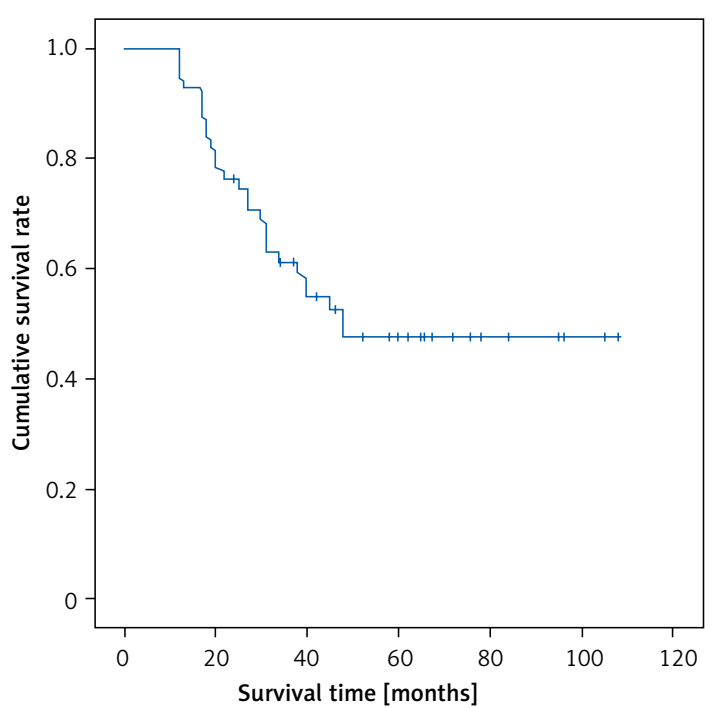

Group $\neg$ Recurrent HB cases $\rightarrow$ Recurrent HB cases-lost

Figure 1. The overall survival curve of the 55 recurrence cases

currence alone group, liver recurrence alone group, liver and lung recurrence group, and recurrence in other sites group. The prognoses of recurrence in different sites were compared. The survival time of the four groups was $84.88 \pm 8.18$ months, 34.67 \pm 4.73 months, $45.02 \pm 5.95$ months, and 37.50 \pm 7.31 months, respectively; the 3 -year survival rates of the 4 groups were $75 \%, 33.3 \%, 51.9 \%$, and $42.9 \%$, respectively. The differences between the 4 groups were statistically significant $\left(\chi^{2}=8.105\right.$, $p=0.044)$, and the lung recurrence alone group had the best prognosis. The prognosis of the liver recurrence alone was the worst group. Four cases of the

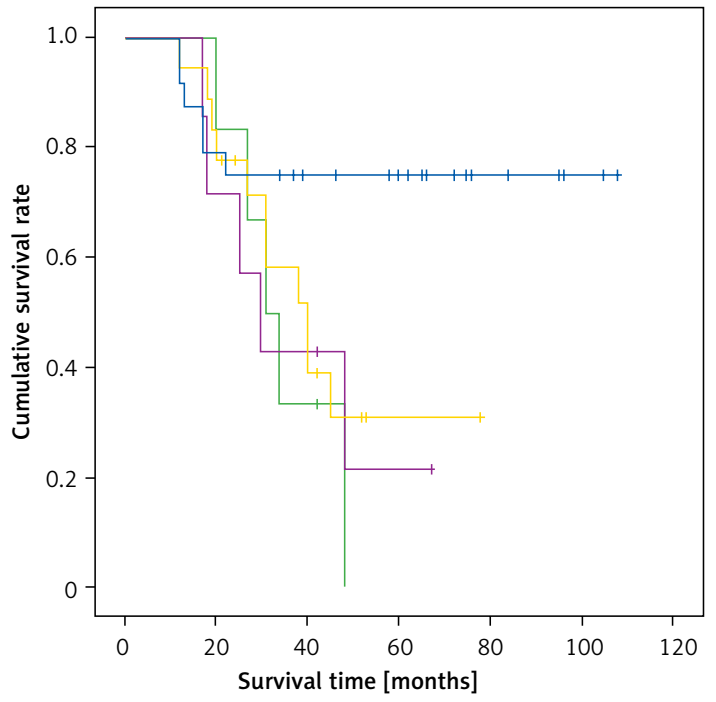

Group $\neg$ Recurrent of lung $\neg$ Recurrence of liver

$\neg$ Both liver and lungs recurrences $\neg$ Recurrence in other sites

+ Lung recurrence-deletion + Liver recurrence-deletion

Recurrence in liver and lungs-deletion

+ Other sites recurrence-deletion

Figure 3 . The overall survival curve of the 4 groups with different sites of recurrence

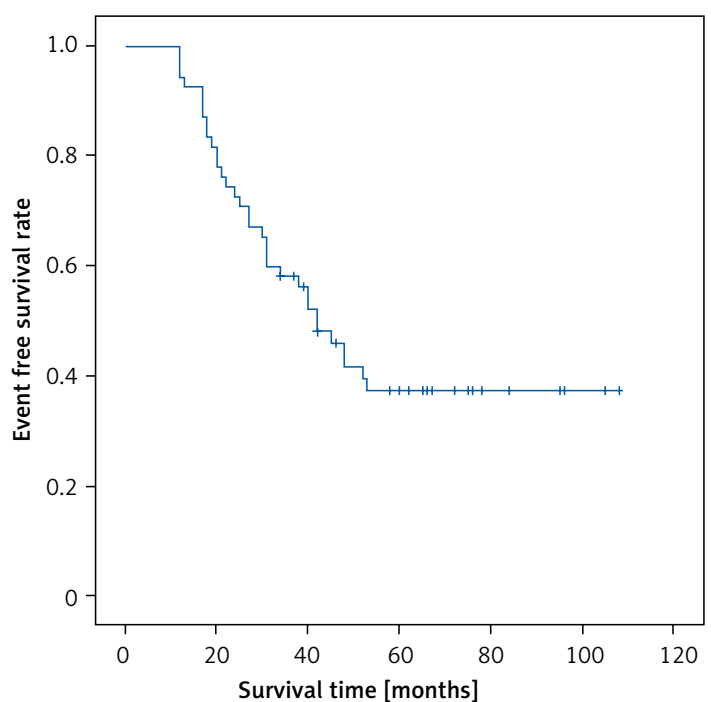

Group $\rightarrow$ Recurrent HB cases + Recurrent HB cases-delete

Figure 2. The event-free survival curve of the 55 recurrence cases

group died due to recurrence, 1 case died of a second tumour, and 1 case died of respiratory failure (Figure 3). The event-free survival time and 3-year eventfree survival rates of the 4 groups were $84.88 \pm 8.18$, $33.67 \pm 4.14$, and $38.23 \pm 4.69,35.29 \pm 6.36$ months, and $75 \%, 33.3 \%, 44.4 \%$, and $28.6 \%)$, with statistical difference $\chi^{2}=14.600, p=0.002$ (Figure 4).

\section{Prognosis of different treatments after recurrence}

After recurrence, 41 patients were treated with chemotherapy and surgery; these pa-

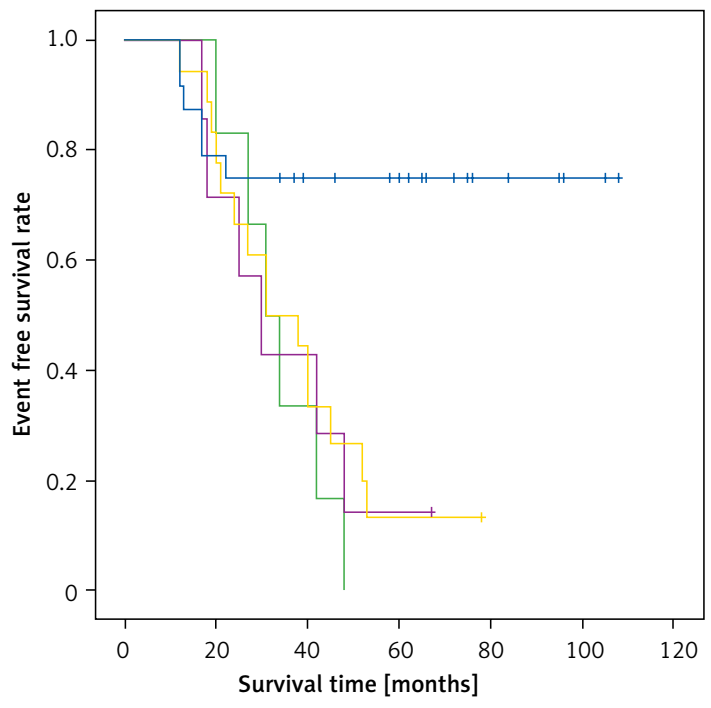

Group $\neg$ Recurrent of lung $\neg$ Recurrence of liver Both liver and lungs recurrences $\neg$ Recurrence in other sites + Recurrence of lung - delete + Recurrence of liver - delete - Both liver and lung recurrence - delete + Recurrence in other sites - deletion

Figure 4. The event-free survival curve of the 4 groups with different sites of recurrence 
tients were regarded as the combined treatment group. Fourteen cases were treated with chemotherapy alone and were regarded as the chemotherapy alone group. The survival time of the combined treatment group was $78.04 \pm 6.13$ months, and the survival time of the chemotherapy alone group was $22.97 \pm 3.17$ months. The 3-year overall survival rates were $75.2 \%$ and $17.1 \%$, respectively. The difference between these 2 groups was statistically significant $\left(\chi^{2}=28.441, p<0.001\right)$, and the prognosis of the combined treatment group was significantly better than that of the chemotherapy alone group (Figure 5). The event-free survival time of the chemotherapy group and combined treatment group was $(32.63 \pm 4.34$ months vs. 55.04 \pm 4.10 months, respectively), and the 3 -year event-free survival rate was $(55.7 \%$ vs. $70 \%$, respectively), and there was a significant difference between the two groups $\left(\chi^{2}=3.328, p=\right.$ 0.0068) (Figure 6).

Of the 24 cases of lung recurrence alone, 5 patients were treated with chemotherapy alone, and 19 patients were treated with chemotherapy combined with surgery during initial treatment. The survival time and overall 3-year survival rate of these 2 groups were $16.20 \pm 1.77$ months vs. $102.95 \pm 4.92$ months and $0 \%$ vs. $94.7 \%$, respectively. The differences between these 2 groups were statistically significant $\left(\chi^{2}=22.107, p<\right.$ $0.001)$. The combined treatment for lung recurrence alone had the best prognosis, and the overall 3-year survival rate was $94.7 \%$. The survival curves are shown in Figure 7.

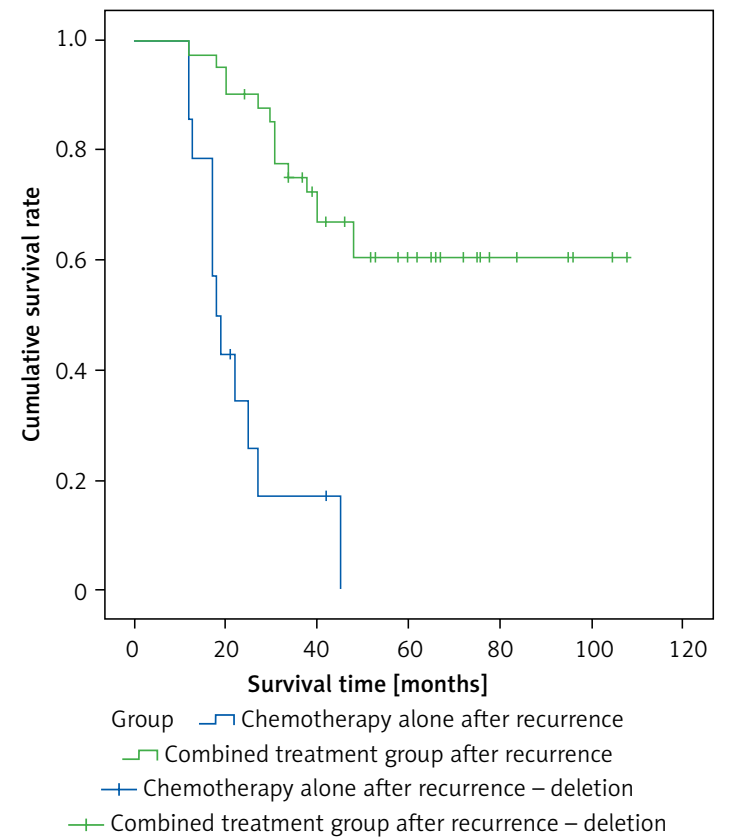

Figure 5 . The overall survival curve of the 2 groups with different treatment after recurrence

\section{Prognosis analysis of different pathological types}

Of the 55 cases of recurrent $\mathrm{HB}, 37$ patients had epithelial type $\mathrm{HB}$, and 15 of these patients died. Eighteen patients had mixed epithelial and mesenchymal type HB, and 12 of them died. The epithelial type survival time was $73.93 \pm 6.82$ months, and the survival time of mixed epithelial and mesenchymal type was $41.31 \pm 6.54$ months. The overall 3-year survival rates were $64.8 \%$ and $46.3 \%$, respectively. The difference between these 2 groups was statistically significant $\left(\chi^{2}=4.672\right.$, $p=0.031$ ) (Figure 8). The event-free survival time and 3-year event-free survival rate were 66.60 \pm 6.66 months vs. $38.01 \pm 5.95$ months; $46.1 \%$ vs. $38.9 \%$, respectively; there was a significant difference between the 2 groups $\left(\chi^{2}=5.162, p=0.023\right)$ (Figure 9). The prognosis of epithelial type was better than that of mixed epithelial and mesenchymal type.

\section{Prognosis and interval from CR to the first recurrence}

The patients were divided into 2 groups based on the interval from $C R$ to the first recurrence: $a<6$ months group and $a \geq 6$ months group. The survival time and total 3-year survival rate of the 2 groups were $60.60 \pm 6.33$ months vs. 80.58 \pm 9.38 months and $53.2 \%$ vs. $87.5 \%$, respectively. The differences between the 2 groups were not statistically significant $\left(\chi^{2}=3.173, p=0.075\right)$ (Figure 10). The event-free survival time and 3-year event-free survival rate of the 2 groups were 52.88

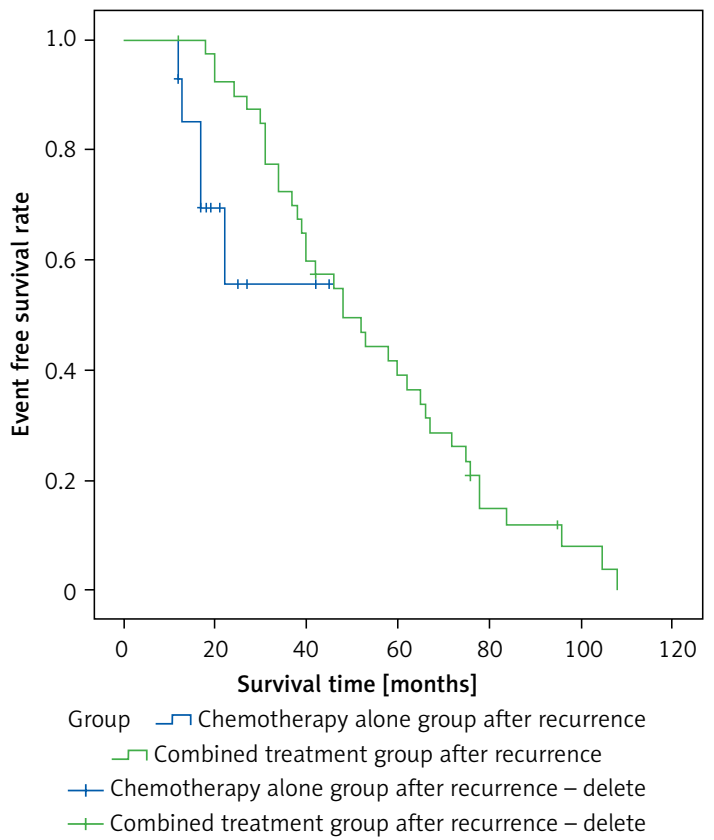

Figure 6 . The overall survival curve of the 2 groups with different treatment after recurrence 


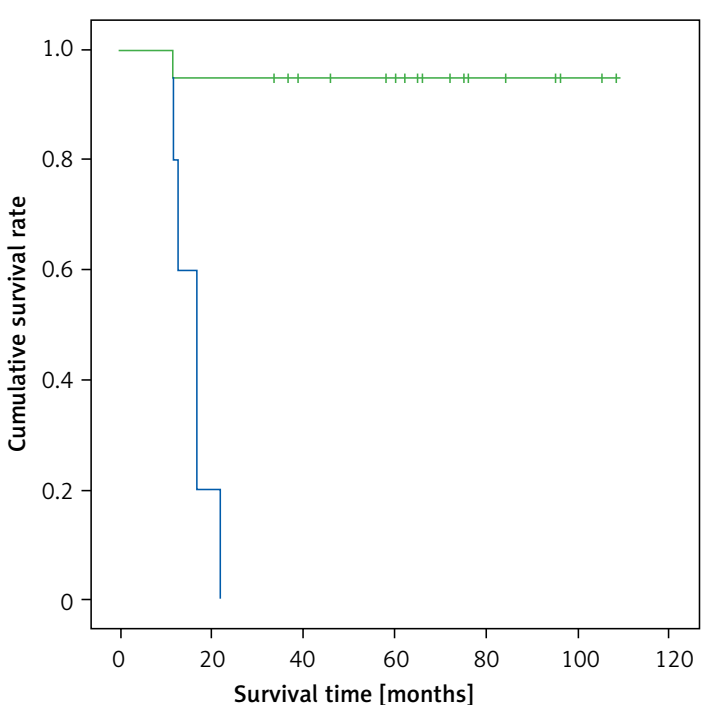

Group $\neg$ Lung recurrence alone and chemotherapy alone $\neg$ Combined treatment group alone for pulmonary recurrence + Pulmonary recurrence alone chemotherapy alone group - deletion

+ Combined treatment group alone for pulmonary recurrence deletion

Figure 7. The overall survival curve of 2 different treatment groups with lung recurrence alone

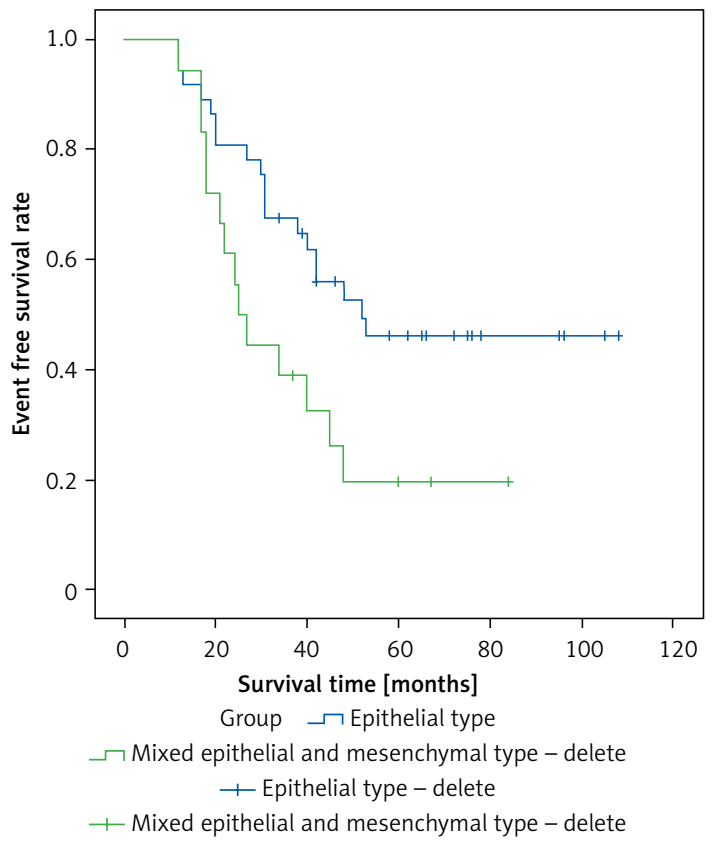

Figure 9. The event-free survival curve of epithelial type and mixed epithelial and mesenchymal type

\pm 5.72 months vs. $80.58 \pm 9.38$ months and $50 \%$ vs. $87.5 \%$, with statistical difference between the 2 groups $\left(\chi^{2}=4.776, p=0.029\right)$ (Figure 11$)$.

\section{Analysis of other factors and prognosis}

The results of the age of onset, gender, PRETEXT classification, COG stage, serum AFP level at the time of first recurrence, and the recurrence fre-

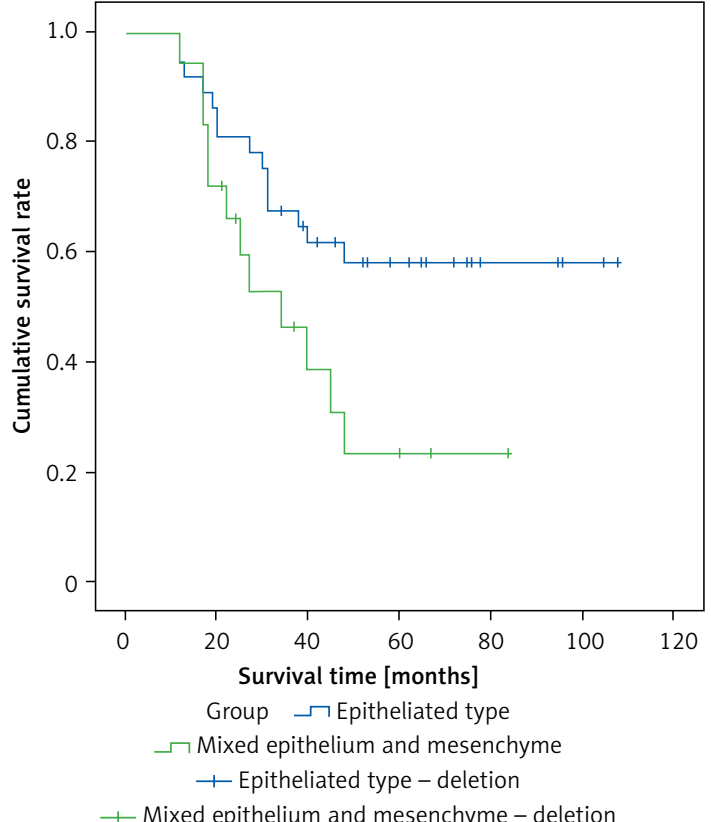

Figure 8. The overall survival curve of epithelial type and mixed epithelial and mesenchymal type

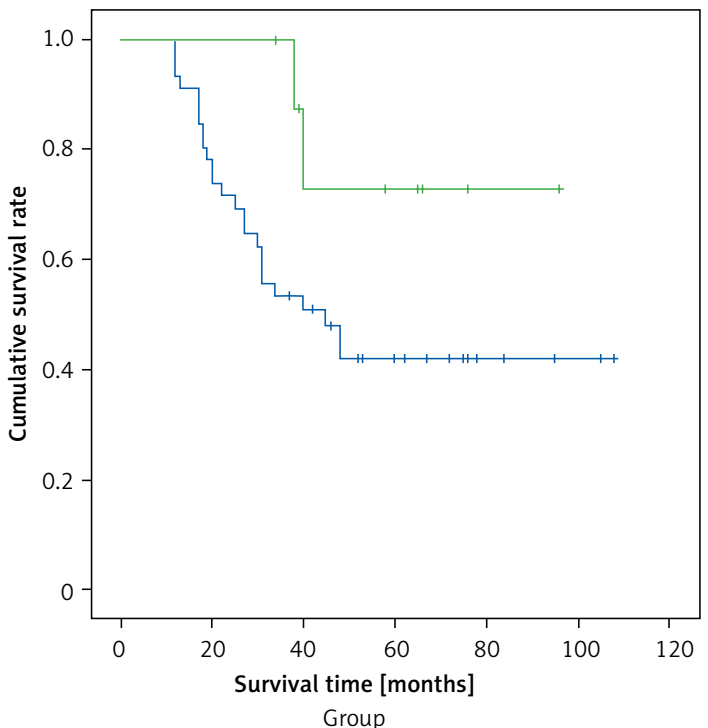

$\neg$ The interval from CR to the first recurrence $<6$ months group $\neg$ The interval from CR to the first recurrence $\geq 6$ months group

+ The interval from $C R$ to the first recurrence $<6$ months group - delete

+ The interval from $C R$ to the first recurrence $\geq 6$ months group - delete

Figure 10. The overall survival curve of different interval time from CR to the first recurrence

quency on prognosis are shown in Table II. There was no statistical difference between all factors on prognosis.

Four factors influencing prognosis (recurrence site, pathological classification, the interval from $C R$ to the first recurrence, and treatment after recurrence) were included in the Cox regression equation. The overall results of the model were: $\chi^{2}$ 


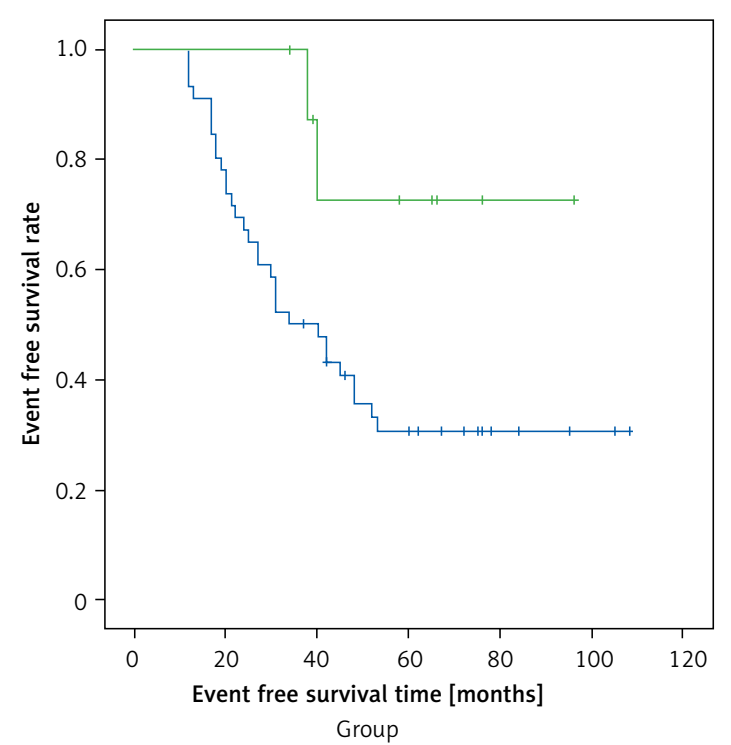

$\neg$ The interval from CR to the first recurrence $<6$ months group

$\neg$ The interval from CR to the first recurrence $\geq 6$ months group

+ The interval from CR to the first recurrence $<6$ months group - delete

+ The interval from CR to the first recurrence $\geq 6$ months group - delete

Figure 11. The event-free survival curve of different interval time from CR to the first recurrence
$=27.710, p<0.001$, and the regression equation showed a statistical significance. Treatment after recurrence was included in the regression equation when other factors remained unchanged. The risk of death was 0.155 times higher in the relapsed patients treated with chemotherapy alone than in the patients who underwent combined treatment $(R R=0.155,95 \% \mathrm{Cl}$ : 0.06-0.399). The results of the Cox regression analysis are shown in Table III.

\section{Discussion}

As is known, the classic risk factors of $\mathrm{HB}$ treatment failure are as follows: PRETEXT stage IV, distant metastasis, older age at the time of diagnosis, low AFP levels, vascular involvement, and the type of small cell undifferentiated pathologically [6]. Of the 55 recurrent cases reported in our study, local recurrence of the liver was found in $24(43.63 \%)$ cases, pulmonary metastasis and recurrence were found in $42(76.36 \%)$ cases, brain metastasis and recurrence was found in $6(10.91 \%)$ cases, and recurrence in the mediastinum was found in $1(1.82 \%)$ case. These are similar to the recurrence sites reported in the literature; however, the pro-

Table II. Results of single-factor analysis affecting prognosis for HB relapse

\begin{tabular}{|c|c|c|c|c|}
\hline Factor & Group & Number of deaths/total & $\chi^{2}$ & $P$-value \\
\hline \multirow[t]{2}{*}{ Age } & $\leq 3$ years & $18 / 38$ & 0.033 & 0.857 \\
\hline & $>3$ years & $9 / 17$ & & \\
\hline \multirow[t]{2}{*}{ Gender } & Male & $20 / 35$ & 2.573 & 0.109 \\
\hline & Female & $7 / 20$ & & \\
\hline \multirow{2}{*}{$\begin{array}{l}\text { PRETEXT Stage at initial } \\
\text { diagnosis }\end{array}$} & Stage III & $3 / 11$ & 1.458 & 0.227 \\
\hline & Stage IV & $24 / 44$ & & \\
\hline \multirow{2}{*}{$\begin{array}{l}\text { COG Stage at initial } \\
\text { diagnosis }\end{array}$} & Stage III & $11 / 23$ & 0.519 & 0.471 \\
\hline & Stage IV & $16 / 32$ & & \\
\hline \multirow[t]{2}{*}{ AFP value at relapse } & $<100 \mathrm{ng} / \mathrm{ml}$ & $0 / 1$ & 0.768 & 0.381 \\
\hline & $\geq 100 \mathrm{ng} / \mathrm{ml}$ & $27 / 54$ & & \\
\hline \multirow[t]{2}{*}{ Recurrence times } & Relapse once & $13 / 28$ & 0.001 & 0.971 \\
\hline & Repeated recurrence & $14 / 27$ & & \\
\hline
\end{tabular}

Table III. COX regression analysis results

\begin{tabular}{|lcccc|}
\hline Influence factor & P-value & RR & \multicolumn{2}{c|}{$95 \% \mathrm{Cl}$} \\
\cline { 3 - 5 } & & & Low value & High value \\
\hline Recurrence of different sites & 0.133 & 1.335 & 0.916 & 1.947 \\
\hline Pathological type & 0.794 & 0.886 & 0.359 & 2.190 \\
\hline $\begin{array}{l}\text { Time to complete remission to the first } \\
\text { recurrence }\end{array}$ & 0.689 & 1.136 & 0.609 & 2.117 \\
\hline Treatment after recurrence & $<0.001^{*}$ & 0.155 & 0.060 & 0.399 \\
\hline
\end{tabular}


portion of cases with pulmonary recurrence and central nervous system recurrence was higher than in previous reports in the literature [14]. In the present study, the prognoses of the 55 cases were analysed according to the site of recurrence. The results showed that the lung recurrence alone group had improved survival, with $75 \%$ surviving at 20 months following diagnosis of relapse. The prognosis of this group given combined treatment was better than that of the chemotherapy alone group. In our retrospective study, the results of the Cox regression analysis revealed that treatment was the only high-risk factor for the prognosis of patients with recurrent HB. In the present study, the overall 3-year survival rate of patients was $75.2 \%$ after combined treatment with surgery and chemotherapy. This figure is significantly higher than the numbers reported in the previous literature [14]. The interval of recurrence time of this group is shorter than that reported in other literature, which may be because there were 24 cases of liver recurrence, and PRETEXT stage IV accounts for the majority of this group. It is thought that, in this retrospective analysis study of a single centre, there were more cases of pulmonary metastasis, and 41 patients were treated with chemotherapy combined with surgery.

Meyers et al. reported that 20 patients with pulmonary relapse had salvage chemotherapy; 13 also had thoracotomy and pulmonary metastasectomy or thoracotomy and biopsy. Only 4 of 13 were long-term survivors. Hence, the review suggests that thoracotomy should be used cautiously in the management of pulmonary relapse and perhaps more aggressively in the management of metastases present at diagnosis and which persist after neoadjuvant chemotherapy [24]. However, Shi et al. analysed and summarized 10 cases of HB with pulmonary recurrence alone reported in the literature from 2005 to 2014, and their results revealed that all 10 cases were treated with lung tumour resection. The median follow-up time of 8 cases was 18.5 months, and all cases achieved CR (8/10, $80 \%)$. Two cases died due to metastasis and recurrence of other sites in the body [15]. There are other reports showing that the combined treatment with chemotherapy and surgery were the main therapy for pulmonary metastasis [25, 26]. The prognosis of patients with pulmonary recurrence alone was good following surgery. In our study, the overall 3-year survival rate of patients in the lung recurrence alone group was $94.7 \%$ after combined treatment with surgery and chemotherapy. The prognosis was better than that reported in the literature [15], which is thought to be related to the large number of patients who underwent pulmonary surgery.
A review of studies on COG I and II did not identify promising drugs for the treatment of recurrent HB [13]. Therefore, it is urgent that an effective therapeutic regime is found. In the present study, the overall 3-year survival rate of the relapsed patients receiving chemotherapy only was $17.1 \%$. Chemotherapy is recommended for patients with pulmonary recurrence first; if there are still residual lesions after chemotherapy, the tumours can be surgically removed [15]. In the present study, a case of multiple recurrences in the lung was treated with routine chemotherapy, bevacizum$a b$, and surgery. The effect of this comprehensive treatment for this case was good, and CR was achieved. The review reported that there were 2 cases with more than 4 pulmonary metastases, who received CR. And after chemotherapy alone, there were 4 nodules that reappeared at the location of the treated nodule, compared to none after resection [15].

Liver transplantation should be considered for patients as followed [27-29]. After neoadjuvant chemotherapy, the patients are assessed as POSTTEXT stage IV, or POST-TEXT stage III accompanied with hepatic veins or inferior vena cava, or patients with blood vessel involvement and liver surgery will affect the blood supply of the remaining liver. There are no extrahepatic lesions and distant metastases after surgical resection or chemotherapy. For children with extrahepatic lesions at the time of diagnosis, if the lesions are completely removal, liver transplantation is also feasible.

Although the overall survival has improved recently [30], the 5-year survival rate is related to the staging and site of recurrence. In the present study, the overall 3-year survival rate of patients with liver recurrence alone was only $33.3 \%$, which was significantly lower than the prognosis of patients with pulmonary recurrence. All patients with liver recurrence did not receive liver transplantation in the present study. Other classic factors affecting prognosis were analysed and compared. The results showed that both the recurrence interval and the pathological classification had a significant effect on prognosis. A Cox regression analysis revealed that these 2 factors were not high-risk factors for prognosis; therefore, the sample size should be expanded to clarify the importance of prognosis evaluation. A statistical analysis of age, gender, recurrence frequency, and AFP level at recurrence on prognosis revealed no significant effect on prognosis.

This article is a retrospective analysis. Most of the patients admitted to our hospital when the treatment effect in other hospitals was not good after recurrence. Some patients were first seen in our hospital after relapse. This will lead to small 
errors in chemotherapy regimens and efficacy evaluations.

In conclusion, different recurrence sites of $\mathrm{HB}$ have different prognoses. In this study, although the group with lung recurrence alone had the best prognosis, the prognosis of patients treated with chemotherapy combined with surgery after recurrence was good, and patients with a longer interval from CR to the first recurrence also had a good prognosis using univariate analysis. However, the results of the Cox regression analysis show that combined treatment was the main risk factor affecting prognosis. Early detection of recurrence and combined treatment with chemotherapy and surgery can achieve CR and improve prognosis.

\section{Acknowledgments}

Wei-ling Zhang and Hui-min Hu contributed equally to this study.

Fund Program: "Climbing the Peak" Talent Plan of Beijing Municipal Administration of Hospital (No. DFL20180201).

\section{Conflict of interest}

The authors declare no conflict of interest.

\section{References}

1. Herzog CE, Andrassy RJ, Eftekhari F. Childhood cancers: hepatoblastoma. Oncologist 2000; 5: 445-53.

2. Turcotte LM, Spector LG. What do we know about the etiology of hepatoblastoma? Hepat Oncol 2014; 1: 7-10.

3. Turcotte LM, Georgieff MK, Ross JA, et al. Neonatal medical exposures and characteristics of low birth weight hepatoblastoma cases: a report from the Children's Oncology Group. Pediatr Blood Cancer 2014; 61: 2018-23.

4. Tanimura M, Matsui I, Abe J, et al. Increased risk of hepatoblastoma among immature children with a lower birth weight. Cancer Res 1998; 58: 3032-5.

5. McLaughlin CC, Baptiste MS, Schymura MJ, Nasca PC, Zdeb MS. Maternal and infant birth characteristics and hepatoblastoma. Am J Epidemiol 2006; 163: 818-28.

6. Haeberle B, Rangaswami A, Krailo M, et al. The importance of age as prognostic factor for the outcome of patients with hepatoblastoma: analysis from the Children's Hepatic tumors International Collaboration (CHIC) database. Pediatr Blood Cancer 2020; 67: e28350.

7. Semeraro M, Branchereau S, Maibach R, et al. Relapses in hepatoblastoma patients: clinical characteristics and outcome: experience of the International Childhood Liver Tumour Strategy Group (SIOPEL). Eur J Cancer 2013; 49: 915-22.

8. Perilongo G, Malogolowkin M, Feusner J. Hepatoblastoma clinical research: lessons learned and future challenges. Pediatr Blood Cancer 2012; 59: 818-21.

9. Trobaugh-Lotrario AD, Katzenstein HM. Chemotherapeutic approaches for newly diagnosed hepatoblastoma: past, present, and future strategies. Pediatr Blood Cancer 2012; 59: 809-12.

10. Towbin AJ, Meyers RL, Woodley H, et al. 2017 PRETEXT: radiologic staging system for primary hepatic malig- nancies of childhood revised for the Paediatric Hepatic International Tumour Trial (PHITT). Pediatr Radiol 2018; 48: 536-54.

11. Czauderna P, Haeberle B, Hiyama E, et al. The Children's Hepatic tumors International Collaboration (CHIC). Novel global rare tumor database yields new prognostic factors in hepatoblastoma and becomes a research model. Eur J Cancer 2016; 52: 92-101.

12. Meyers RL, Maibach R, Hiyama E, et al. Risk-stratified staging in paediatric hepatoblastoma: a unified analysis from the Children's Hepatic tumors International Collaboration. Lancet Oncol 2017; 18: 122-31.

13. Trobaugh-Lotrario AD, Feusner JH. Relapsed hepatoblastoma. Pediatr Blood Cancer 2012; 59: 813-7.

14. Trobaugh-Lotrario AD, Meyers RL, Feusner JH. Outcomes of patients with relapsed hepatoblastoma enrolled on Children's Oncology Group (COG) phase I and II studies. J Pediatr Hematol Oncol 2016; 38: 187-90.

15. Shi Y, Geller JI, Ma IT, et al. Relapsed hepatoblastoma confined to the lung is effectively treated with pulmonary metastasectomy. J Pediatr Surg 2016; 51: 525-9.

16. Matsunaga T, Sasaki F, Ohira M, et al.; Japanese Study Group for Pediatric Liver Tumor. Analysis of treatment outcome for children with recurrent or metastatic hepatoblastoma. Pediatr Surg Int 2003; 19: 142-6.

17. Malogolowkin MH, Katzenstein HM, Krailo M, et al. Redefining the role of doxorubicin for the treatment of children with hepatoblastoma. J Clin Oncol 2008; 26: 2379-83.

18. Pediatric Oncology Professional Committee of Chinese Anti-cancer Association, Pediatric Surgery Branch of Chinese Medical Association. Multidisciplinary expert consensus on diagnosis and treatment of pediatric hepatoblastoma (CCCG-HB-2016). Chin J Pediatr Surg 2017; 38: 733-9.

19. López-Terrada D, Alaggio R, de Dávila MT, et al.; Children's Oncology Group Liver Tumor Committee. Towards an international pediatric liver tumor consensus classification: proceedings of the Los Angeles COG liver tumors symposium. Mod Pathol 2014; 27: 472-91.

20. Tanaka Y, Inoue T, Horie H. International pediatric liver cancer pathological classification: current trend. Int J Clin Oncol 2013; 18: 946-54.

21. Roebuck DJ, Aronson D, Clapuyt P, et al.; International Childrhood Liver Tumor Strategy Group. 2005 PRETEXT: a revised staging system for primary malignant liver tumours of childhood developed by the SIOPEL group. Pediatr Radiol 2007; 37: 123-32.

22. Meyers RL, Rowland JR, Krailo M, et al. Predictive power of pretreatment prognostic factors in children with hepatoblastoma: a report from the Children's Oncology Group. Pediatr Blood Cancer 2009; 53: 1016-22.

23. Brown J, Perilongo G, Shafford E, et al. Pretreatment prognostic factors for children with hepatoblastoma: results from the International Society of Paediatric Oncology (SIOP) study SIOPEL 1. Eur J Cancer 2000; 36: 1418-25.

24. Meyers RL, Katzenstein HM, Krailo M, et al. Surgical resection of pulmonary metastatic lesions in children with hepatoblastoma. J Pediatr Surg 2007; 42: 2050-6.

25. Hishiki T, Watanabe K, Ida K, et al. The role of pulmonary metastasectomy for hepatoblastoma in children with metastasis at diagnosis: results from the JPLT-2 study. J Pediatr Surg 2017; 52: 2051-5.

26. Zsíros J, Maibach R, Shafford E, et al. Successful treatment of childhood high-risk hepatoblastoma with dose-intensive multiagent chemotherapy and surgery: 
final results of the SIOPEL-3HR study. J Clin Oncol 2010; 28: 2584-90.

27. Otte JB, Pritchard J, Aronson DC, et al.; International Society of Pediatric Oncology (SIOP). Liver transplantation for hepatoblastoma: results from the International Society of Pediatric Oncology (SIOP) study SIOPEL-1 and review of the world experience. Pediatr Blood Cancer 2004; 42: 74-83.

28. Reyes JD, Carr B, Dvorchik I, et al. Liver transplantation and chemotherapy for hepatoblastoma and hepatocellular cancer in childhood and adolescence. J Pediatr 2000; 36: 795-804.

29. Austin MT, Leys CM, Feurer ID, et al. Liver transplantation for childhood hepatic malignancy: a review of the United Network for Organ Sharing (UNOS) database. J Pediatr Surg 2006; 41: 182-6.

30. Yang T, Whitlock RS, Vasudevan SA. Surgical management of hepatoblastoma and recent advances. Cancers 2019; 11: 1944 